\title{
CRISPR/Cas9 System: A Breakthrough in Genome Editing
}

\section{Asad Razzaq* and Ardas Masood}

Institute of Molecular Biology and Biotechnology, Bahauddin Zakariya University, Multan, Pakistan

\begin{abstract}
Clustered Regularly Interspaced Short Palindromic Repeats is a new and advance gene editing tool using specific nuclease enzyme for specific cleavage. It is the most efficient technique, commonly used for many purposes like gene therapy, production of desired plants and transgenic animals. But it has some limitations like off-target issue and this issue can be minimized by the production of specific sequence of guide RNA. In the future, it can be used for the treatment of many human genetic diseases.
\end{abstract}

Keywords: Gene expression; Double-strand break; Gene therapy

Abbreviations: TALEN: Transcription Activator-Like Effector Nucleases; ZFN: Zinc Finger Nuclease; ZFP: Zinc Finger Peptide; PAM: Protospacer Adjacent Motif; HDR: Homologous Directed Repair; NHEJ: Non-Homologous End Joining; CRISPR: Clustered Regularly Interspaced Palindromic Repeats

\section{Introduction}

Genome editing is an insertion, deletion or replacement of a gene for eliminating or inducing specific and desired characters in genome. In the past, it's totally a dream that specific or mutated gene can be deleted from genome and foreign or desired gene can be inserted into genome. But this dream was true in 1960 when the cell lines developed [1]. In the Mid 1960s, researchers work on SV40 transformed cells of viral DNA and reported that virus also has the ability to transfer genes into any type of target cell [2,3]. Stanfield et al. [4] used virus for first time to transfer gene for the treatment of a girl who was suffered with hyperargininemia but they failed to accomplish any result.

Now days, genome editing is easiest and most accurate due to knowing the sequence and functions of DNA and nucleases [5]. Genome editing technique was first use in 1990 by the chemist, Kim et al. [6]. Three genome editing techniques are present that are commonly used. First is Zinger Finger Nuclease (ZFNs) second is Transcription Activator-Like Effector Nucleases (TALENs) and the third is Clustered Regularly Interspaced Short Palindromic Repeats with Cas9 Nuclease (CRISPR/Cas9). All these techniques have two domains for perfect and accurate genome editing. Specifically, the first is specific DNA binding domains that are complementary to the target sequence and second is nuclease domain that produces the Double Strands Break (DSBs). ZFN and TALEN use chimeric protein as a DNA binding domains whereas CRISPR/Cas system uses specific sequence of RNA molecule for DNA binding instead of chimeric protein [7].

ZFN is a first technique for genome editing. It contains two domains as write earlier. First is Zinc Finger Peptide (ZFP) domain for DNA binding and second is FokI restriction enzyme for cleaving DNA strands. Binding domain binds to the complementary sequence of target DNA and then FokI restriction enzyme cleaves the double strand of DNA on site where ZFP and DNA bind [8]. But the problem is its specificity is very low due to the presence of the tandem array of Cys2His2 zinc fingers in ZFP region because each finger recognizes only 3 bp $[6,8]$. ZFNs are used to correct the mutations of genes that cause sickle cell anemia and Hemophilia B [9].

TALEN is the second technique that is commonly used for genome editing. It comes from the bacterium Xanthomonas a pathogenic plant. First it is detected in 2010 and it also has two domains. First is transcription activator-like effector domain for binding with DNA. It is a conserved region of 30-35 repeated amino acids. And second domain is FokI restriction enzyme for DNA Cleavage [7,10,11]. TALEN is economical and their manufacturing is easy as compared to ZFNs [12]. TALENs also have the ability to do disease modeling [13].

CRISPR-Cas technique is the most common and advanced technique for genome editing. It is an accurate method for gene modification and it also has the ability to edit more than one gene at a time $[14,15]$. This system is based on adaptive immune system of bacteria. It was initially noticed in bacteria in 1987, found in $40 \%$ of the sequenced bacterial genome and almost $80 \%$ of the sequenced genome of archaea $[16,17]$. Researchers use this system first time in $2002[17,18]$. CRISPR-Cas system also has two domains like others, first is Single Guide RNA (sgRNA) that is used for specific binding purpose and second is Cas9 enzyme that is used for cleavage purpose. sgRNA contains two types of RNAs, CRISPR RNA (crRNA) and Trans-activating crRNA (tracrRNA). So, those gRNA that have both RNA (crRNA and tracrRNA) are sufficient for perfect genome editing [15]. sgRNA have a specific sequence that is complementary to target sequence so, specificity depends on the sequence of gRNA [19]. Cas9 is an enzyme that has a properties of helicases and polymerases and it is used for the Double Strand Breaks (DSBs) $[16,20]$. Cas9 make a complex with sgRNA (crRNA and tracrRNA) and cleaves the DNA sequence that is complementary to the sgRNA sequence and it was first identified in 2001 [21,22].

CRISPR locus is basically a repeated sequence of conserved region interspaced by a spacer sequence that is non-repeated sequence. Spacer is a viral DNA that is cleaved by a Cas9 enzyme and it is joined with a CRISPR locus as a spacer $[15,23]$. Downstream of a spacer called Protospacer that have a specific sequence called Protospacer Adjacent Motif (PAM). PAM is simple and 2-5 bp long. Cas9 recognizes the PAM sequence on viral DNA and if PAM is not found then it not cleaved the DNA. Usually the sequence of PAM is 5'-NGG-3'. 'N' is any nucleotide base and ' $G$ ' mean Guanine. Cleavage occurs only on that side where PAM sequence present [14,24]. In 2005, researcher have successfully constructed the spacer sequence from phage genome [25,26]. Almost 40 types of Cas proteins discovered but the most common is Cas9 that is produced in Streptococcus pyogenes [23,27,28].

*Corresponding author: Asad Razzaq, Institute of Molecular Biology and Biotechnology, Bahauddin Zakariya University, Multan, Pakistan, Tel: +923064730096; E-mail: asadrazzaqlahore@gmail.com

Received March 23, 2018; Accepted April 10, 2018; Published April 17, 2018

Citation: Razzaq A, Masood A (2018) CRISPR/Cas9 System: A Breakthrough in Genome Editing. Mol Biol 7: 210. doi: 10.4172/2168-9547.1000210

Copyright: ( 2018 Razzaq A, et al. This is an open-access article distributed under the terms of the Creative Commons Attribution License, which permits unrestricted use, distribution, and reproduction in any medium, provided the original author and source are credited. 
CRISPR/Cas system is divided into 8 subtypes [20]. But according to modern classification, it is divided into three types [29]. In type I and III, first pre-crRNA produce and when this pre-crRNA mature then it fuse with Cas9 enzyme to form a complex. Then this complex cleaves the sequence of DNA that is complementary to the sequence of crRNA. Type I and III are not commonly use [22]. While type II is the most famous and it is commonly use in gene editing. In this type, gRNA guide the Cas9 enzyme for producing DSBs $[15,30]$.

Comparing CRISPR/Cas9 system with ZFNs and TALENs, ZFNs and TALENs are time consuming and have more off target effects. While CRISPR/Cas9 system has very less off-target effects because it uses a specific sequence of guide RNA that binds with the specific sequence of DNA (target DNA) and then Cas9 enzyme cleaves the strands where binding occur. This system is able to produce transgenic animal and edit more than one gene (multiplexes gene editing) at a time [31,32] (Table 1).

\section{CRISPR-Cas System of Immunity}

CRISPR-Cas system is based on immune system of bacteria and it has three stages to work as an immune system in bacteria. First is adaptation, second is expression and final and third is interference. During Adaptation stage that is also called recognition stage, viruses or phages attack the bacteria and introduce their own DNA into bacteria. Cas gene recognizes this DNA fragment as a foreign particle and breaks it into small fragments. Then these small fragments of invading DNA are incorporated into CRISPR locus as spacers [23]. At Expression stage, spacer produces a pre-crRNA by acting as a transcriptional template. When pre-crRNA produce then Cas gene perform a series of action and convert pre-crRNA into mature crRNA. In Interference stage, mature crRNA guides the enzyme to the target site and enzyme destroys the invading or foreign DNA $[23,33,34]$.

\section{CRISPR/Cas9 System in Genome Editing}

In gene editing, it is most common because it is efficient in gene editing and has very less off-target effects. So, production of desired characters can be very easy by using this system.

\section{Cas9 Structure and Working Mechanism}

CRISPR/Cas9 is highly efficient, precise, accurate and specific due to Cas9 enzyme. Cas9 is basically a bi-lobed structure having active sites and two grooves for binding of nucleic acid that is REC (large recognition lobe) and NUC (small nuclease lobe). Both are connected by a helix bridge [35-37]. Specificity of Cas9 depends on REC whereas NUC has further two domains. First, RuvC and $\mathrm{HNH}$ and second is
PAM interacting domain (PI). In natural conditions, Cas9 is inactive and it is active only when REC lobe of Cas9 binds with sgRNA. A complex of Cas9 and sgRNA forms and then this complex scan the target DNA sequence and recognize the target site easily because target sequence is complementary to sgRNA sequence and PAM sequence also present on downstream of target site. Then sgRNA bind with target sequence and after binding, target sequence strand are cleave by $\mathrm{HNH}$ and the opposite strand of the target sequence are cleave by RuvC and DSBs are forms [30,38-41]. Researchers have developed an advanced type of CRISPR/Cas9 that can identify 20-24 nucleotides sequence of target site that is complementary to the sgRNA and 2-5 nucleotides of PAM sequence. As a result, CRISPR/Cas9 able to target a sequence which is $22-29$ nucleotides long $[42,43]$.

\section{Repair Processes}

When HNH and RuvC of Cas9 form DSBs then CRISPR/Cas9 system activates the repair mechanism that fixed the DSBs [41]. Two types of repair mechanisms present that play an important role in the fixing of DSBs. First is Homologous Directed Repair (HDR) and second is Non-Homologous End Joining (NHEJ). HDR is an error free method and it joins only homologous templates. Commonly used in plants for gene knock-in, however, it is more complicated as compared to NHEJ [44-46]. While NHEJ is an error prone method, it applies DNA ligase to rejoin the ends. It insert or delete the specific gene sequence and causes Indels mutation. So, it is commonly used for gene knock-out [44,47] and for working of CRISPR/Cas9 system (Figure 1) [48].

\section{Delivery Methods}

Delivery of CRISPR/Cas9 is very critical problem. Accurate delivery can produce an efficient and desired genome editing. Both viral and non-viral based systems are used for the delivery of CRISPR/Cas9 to a target site.

\section{Viral Delivery Method}

Viral systems have been preferred over the past few decades to deliver the gene like lentiviruses, adeno-associated virus (AAV), retroviruses and adenoviruses. Viral delivery systems are divided into two categories. First, those viruses whose genetic material can be integrated into host genetic material, like lentiviruses or retroviruses. Those viruses that remains inside the nucleus and cytoplasm of host like AAV or adenoviruses [49]. Viral delivery has both advantages and disadvantages like retrovirus integrates into the host and activates the oncogene that increases the frequency of haphazard mutations. But the advantage is retroviral delivery system produces a long term expression [50].

\begin{tabular}{|c|c|c|c|}
\hline Factors & ZFN & TALEN & CRISPR/Cas9 \\
\hline Nuclease Assembly & Significant & Significant & Simple \\
\hline Choose Target Site & Limited & Limited & Unlimited \\
\hline Gene Mutation & Limited & Limited & Unlimited \\
\hline Target efficiency & High & Low & Low \\
\hline Components & ZFP + Fokl Fusion Protein & TALE + Fokl Fusion Protein & guide RNA + Cas9 Protein \\
\hline Enzymes & Fokl Restriction Enzyme & Fokl restriction enzyme & Cas9 Protein \\
\hline Time & $7-15$ days & 5-7 days & 1-3 days \\
\hline Cost & High & High & Low \\
\hline Recognition Site & $9-18 b p$ & $14-20 \mathrm{bp}$ & 22 bp (20 gRNA sequence +2 bp of PAM sequence) \\
\hline Immunogenicity & Low & Unknown (protein derived from Xanthomonas sp. & Unknown (protein Derived from Different Bacteria) \\
\hline Ex vivo Delivery & Easy & Easy & Easy \\
\hline Specificity & Low & Moderate & Highly \\
\hline
\end{tabular}

Table 1: Comparing CRISPR/Cas9 system with ZFNs and TALENs, ZFNs and TALENs. 


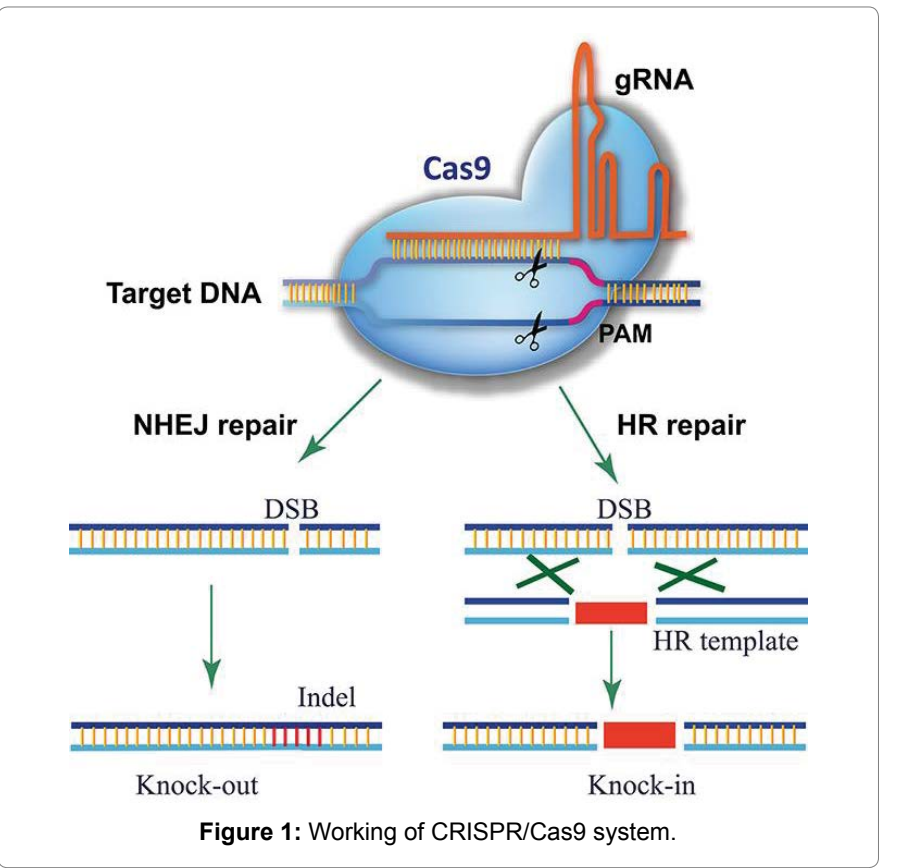

AAV is the approved method for gene therapy because it is safe, produces long term expression and the immune response is mild [51]. Nevertheless, some limitations also present like poor packaging ability $(4.7 \mathrm{~kb})$ while size of Cas 9 of Streptococcus pyogenes is $4.2 \mathrm{~kb}$. So, it creates a challenging situation to deliver SpCas9, sgRNA and additional necessary components by using AAV delivery method [51,52]. This problem is minimized by using small orthologs of Cas9 like SaCas9 $(\sim 3.3 \mathrm{~kb})$ or StCas9 $(\sim 3.3 \mathrm{~kb})$. Still a major problem using these small orthologs of Cas9 is that they need large PAM sequences like SaCas9 identify 5'-NNGRRT-3' while StCas9 identify 5'-NNAGAAW-3' sequences [53-55].

Lentivirus is also use to deliver genes. Researchers modify the HIV1 and make Lentivirus capable to deliver gene into any type of host. Its packaging capacity is high as compared to others $(9.7 \mathrm{~kb})$. So, it easily delivers Cas9, sgRNA and other additional necessary components into host [56].

\section{Non-Viral Delivery Approach}

Non-viral delivery is also applicable for delivery CRISPR/Cas9 to the target sites. In this approach, liposomes, nano-particles and hybrodynamic injections are commonly used [56].

This approach has many advantages:

- It is safe

- Packaging capacity is large

- Immune response is low

- Production is easy
Hybrodynamic injection is the recently use approach for delivery of CRISPR/Cas9. It is high volume injection (approximately 8-10\% of mice body weight). This injection delivers directly into the vascular system of the body of host. Delivery speed is very high (approximately 5-7 s). Researchers use this method or injection and deliver the CRISPR/Cas9 into the liver for study purpose [57,58]. But the problem is it damages the heart and liver tissues so, it is not clinically applicable [59] (Table 2).

\section{New Developments}

The fact that CRISPR/Cas9 is the preferred method used for genetic editing makes it difficult to expand on new developments. The history is short and the system is widely used because of its precision and efficiency in genome editing [60].

\section{Cas9 Modifications}

Qi et al. [61] produced a dCas9 (dcas9 or CRISPRi) that is inactive by producing point mutation in $\mathrm{HNH}$ and RuvC domains of Cas9. dCas9 lacks cleavage activity, so it cannot be able to produce DSBs. This $\mathrm{dCas} 9$ is used for the activation and repression of specific gene. dCas9 first time developed in 2013 at UCSF [60]. Both (dCas9 and sgRNA) are co-expressed in transcriptional elongation so, transcription can be control by CRISPRi and it is able to express or repress any target gene as shown in Figure 2 [62].

\section{Cas9 Combines with Proteins}

dCas9 fuse with epigenetics factors (methylation) and produce desired changes in the epigenomes of host. Cas9 also combined with some fluorescent proteins for labeling purpose. It is also used to study the complex organization of nuclear material [63].

\section{PAM Developments}

New Cas9 have been discovered that able to identify variety of PAM sequences. Like StCas9 recognizes "NNAGAA" while SaCas9 recognizes three different PAM sequences: "NNGAGT" "NNGGGT"

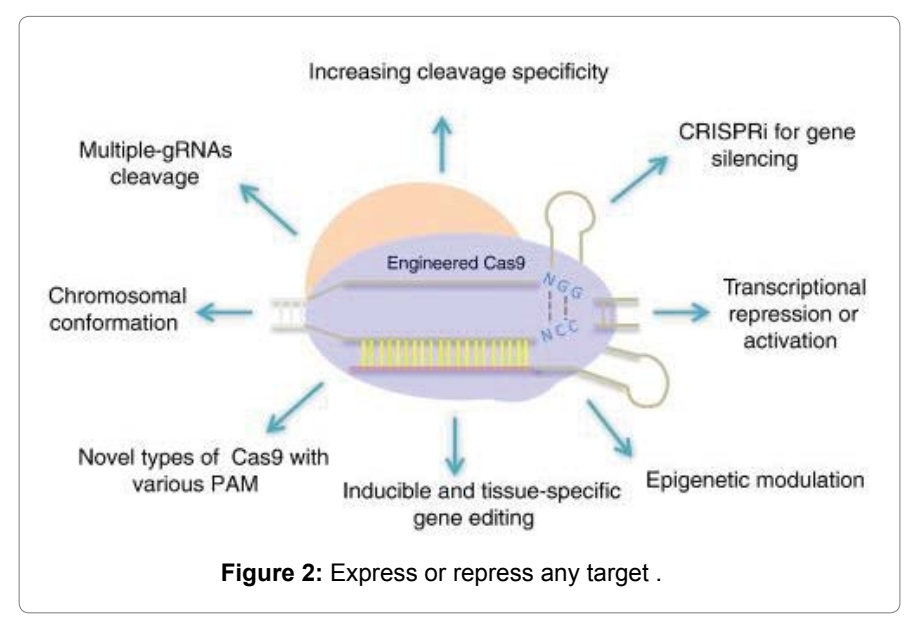

\begin{tabular}{|l|c|c|c|}
\hline Mode of Delivery & Immunogenicity & Duration of Expression \\
\hline Retro-virus & Low & Long term \\
\hline Adenovirus & Low & Medium \\
\hline Lenti-virus & Low & Long term \\
\hline Nano-particles & Under Evaluation & Transient \\
\hline Proteins & Under Evaluation & Transient \\
\hline RNA & Low & Transient \\
\hline
\end{tabular}

Table 2: Hybrodynamic injection process for delivery of CRISPR/Cas9. 
and "NNGAAT" [54,55]. A new enzyme (Cpf1) has been discovered that recognizes T-rich PAM sequences [64].

\section{Cpf1 Proteins}

In 2015, Chen et al. [63] and Zetche et al. [64] discovered a new class of CRISPR protein name Cpf1. It is discovered from Prevotella and Francisella and it reduces the current limitation of Cas9. $16 \mathrm{Cpf1}$ proteins have been identified, but only two are appropriate for human. CRISPR/Cpf1 system has some advantages over CRISPR/Cas9 system for example, it is small in size and easy to handle. Compare with Cas9, Cpf1 requires a single guide RNA while Cas9 requires two guide RNA molecules. It produces sticky ends after cleavage that improves its accuracy by producing 5'overhang while Cas9 produces blunt ends and Cpf1 doesn't needs tracrRNA for its working while Cas9 needs tracrRNA $[14,65]$.

\section{Applications of CRISPR/Cas9 System}

CRISPR/Cas9 is the modern technique for genome editing and it is very simple, efficient and precise method. This system has the ability to do multiplexed gene editing [66]. It also has the ability to change chromosomal target by insertion or deletion (Indels) [67]. This system has a variety of applications like ability to remove internal viral genes from host that causes diseases [68].

\section{Gene Therapy}

CRISPR/Cas9 system has the ability to treat many genetic and viral diseases like Cystic Fibrosis. For cystic fibrosis treatment, first isolate intestinal stem cell and develop it in culture in laboratory and then remove the target allele (F508) and placed back to the human $[69,70]$. Huntington is also treated by CRISPR/Cas9. In this disease treatment, CAG repetitions present in HTT gene that can be removed by CRISPR/ CAS9 [71]. Rett Syndrome and Schizophrenia are also treated by CRISPR/Cas9 system [72,73]. Nowadays, researchers use the CRISPR/ CAS9 system to mutate the oncogene for treating cancer, but it is on trial basis. Edina et al. disturbed the long terminal repeats of HIV-1 that cause AIDS by CRISPR/Cas9 so, the concentration of AIDS in that patients is decreased [74,75].

\section{Biotechnology}

CRISPR/Cas system has also been used in biotechnology and pharmacology. It plays important role in making vaccines for Pseudorabies virus [76]. Many pharmaceutical companies started investing in this field and the aim of the private companies may be achieving high profit.

Bill Lundberg said; "Interest in the gene editing forces of CRISPRCas 9 by pharmaceutical companies literally exploded in the last year" [77].

Now a day, CRISPR-Cas9 can able to treat HIV patients. Hepatitis $\mathrm{B}$ is also treated by CRISPR-Cas9 system. In this disease, covalently closed circular DNA (cccDNA) present that produces a new type of antigen and it can cause hepatitis B. Researchers removes cccDNA from hepatitis B patient's genome by using CRISPR/Cas9 system [78].

\section{In Plants}

This diverse system is also used in plants for many purposes like gene mutation, gene knock-in, gene knock-out, epigenomes modifications, etc.

In Arabidopsis, several genes (AtPDS3, AtFLS2, AtADH, AtFT, AtSPL4, AtBRI1) are targeted by using mutational efficiencies and these mutations are transferred to their offspring successfully [79].
Some other successful examples have been reported in other crops like Sorghum bicolor, Zea mays, Citrus sinensis, Populus tricocarpa, Solanum esculentum and Triticum aestivum [80].

\section{Genome Editing}

Insertion, deletion or replacement of gene from genome is called genome editing. Introduce the desired modifications in genome by mixing multiple gRNA with Cas9, this produces multiple mutations in mammalian genome [30]. CRISPR/Cas9 also show its editing ability in zebra fish [81], mice [31], drosophila [44,82] and bacteria [83]. Bassett et al. [82] apply CRISPR/Cas9 injections directly into the embryo of Drosophila for constructing desired mutagenesis and the success rate is $88 \%$. These mutations can be transferred to the next generation through germ line and their success rate is $33 \%$. This system has the ability to produce desired phenotypes and eliminate disease causing mutations from animal [84].

\section{Transcriptional Regulations}

Researchers produce catalytically inactive Cas9 protein; name dead Case or dCas9, by point mutation in $\mathrm{HNH}$ and RuvC domains of Cas9 enzyme. dCas9 forms a complex with CRISPRi and disturbs the functional sites of transcription that controls the transcription process. Then this complex interferes in the elongation steps of transcription and express or repress the target sequence $[85,86]$. Brief application of CRISPR/Cas9 system [87] (Figure 3).

\section{Limitations of CRISPR/Cas9 system}

$$
\text { CRISPR/Cas9 has following limitations. }
$$

\section{Off-Target Effects}

Off-target effects are major problem in genome editing. ZFNs and TALENs also have this problem. CRISPR/Cas9 also faces this off-target issue, but in this system the quantity of off-target effects are less compared to ZFNs and TALENs. Large genomes have large and complex sequences and this complex genome has some identical or homologous sequences. CRISPR/Cas 9 cleaves these identical sequences instead of target sequence and produced un-desired mutations that are harmful to individual health $[30,40,42]$.

\section{Some Reasons for Undesired Mutations are Identified as Followed}

First, Cas9 and sgRNA ratio is not accurate and high ratio of Cas9 and sgRNA produces more off-target effects $[88,89]$.

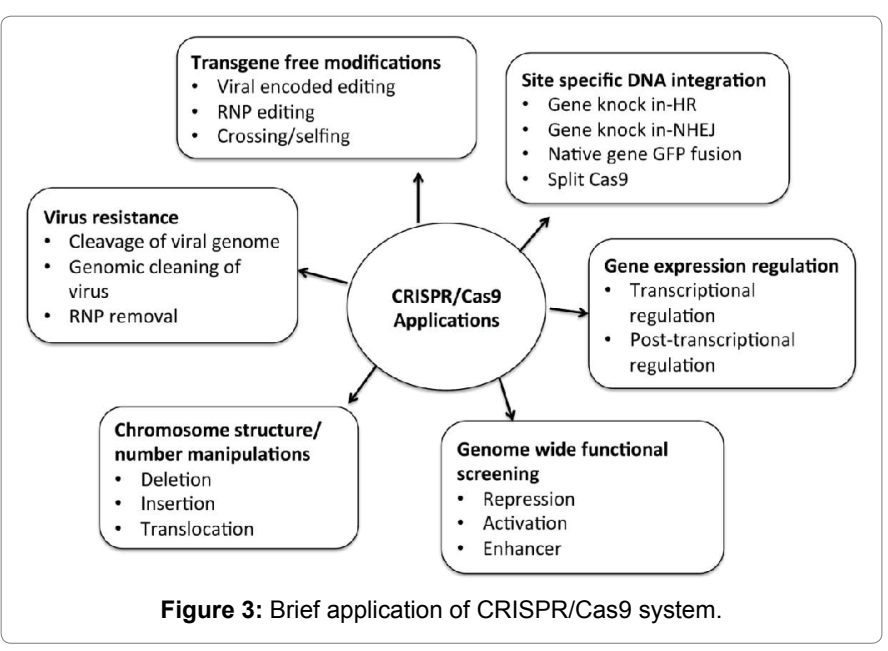


Second, indiscriminate PAM sites also cause off-target effects. So, use bioinformatics tools like E-CRISPR for designing gRNA to overcome this problem $[90,91]$.

Many systems and promoters are present for the expression of Cas9 and sgRNA. Select optimal promoter vectors for the expression of Cas9 and sgRNA. In eudicots, U6 promoter use for sgRNA expression while $35 \mathrm{~S}$ CaMV promoter for Cas9 expression. In monocots different promoters are used for the expression of sgRNA and 35S and Ubi promoters are used for the expression of Cas9 [92]. Some researchers reported an abrupt mutation when using CRISPR/Cas9 system [40].

\section{PAM Dependence}

PAM is a specific sequence present on the downstream of the target sequence. Cas9 recognizes the PAM sequence and when it recognized the PAM sequence then Cas9 cleaves the double strands of DNA. Cas9 is totally depending on PAM sequences because if PAM sequence is not recognized by Cas9 enzyme then it will not able to cleave the DNA double strands. PAM sequence are different for different Cas9 like "NGGNG" and "NNAGAAW" from Streptococcus thermophiles $[93,94]$, "NGG" from Streptococcus pyogenes and "NNNNGATT" from Neisseria meningitides $[95,96]$.

\section{Conclusion}

Good gene editing tools has ability to target any sequence without any off-target effects and the CRISPR/Cas9 is the one. It is simplest, precise and economical method of gene editing as compared to previous ones. It uses a specific sequence of RNA called guide RNA whose sequence is complementary to the target DNA sequence and Cas9 enzyme that act as helicases and polymerases. Specificity is depending on gRNA and Cas9. It has some off-target effects but this problem is in less quantity as compared to previous ones and this issue can be minimized by the production of specific sequence of gRNA. CRISPR/Cas9 uses for many purposes like production of desired, high yield and diseases resistance plants. In animals, it is used to treat animal diseases and produces transgenic animals. In human, use for gene therapy, treatment of genetic and viral diseases, production of vaccines and active or silence any gene sequence.

\section{References}

1. Chang N, Sun C, Gao L, Zhu D, Xu X, et al. (2013) Genome editing with RNAguided Cas9 nuclease in Zebrafish embryos. Nat Publ Gr 23: 465-472.

2. Hill M, Hillova J (1972) Virus recovery in chicken cells tested with rous sarcoma cell DNA. Nat New Biol 237: 35-39.

3. Cells S, Sambrook BJ, Westphal H, Srinivasan PR, Dulbecco R (1968) The integrated state of viral DNA in SV40-transformed cells. Proc Natl Acad Sci 60: 1288-1295.

4. Stanfield R, Lowenthal A, Terheggen HG, Columbo JP (1973) Induction of arginase activity with the shope papilloma virus in tissue culture cells from an argininemic patient. JEM 137: 1091-1096.

5. Urnov FD, Miller JC, Lee YL, Beausejour CM, Rock JM, et al. (2005) Highly efficient endogenous human gene correction using designed zinc-finger nucleases. Nature 435: 646-651.

6. Kim YG, Cha J, Chandrasegaran S (1996) Hybrid restriction enzymes: Zinc finger fusions to Fok I cleavage domain. Proc Natl Acad Sci 93: 1156-1160.

7. Focus $N$ (2013) The CRISPR craze. Science 341: 833-837.

8. Wolfe SA, Nekludova L, Pabo CO (2000) DNA Recognition by $\mathrm{Cys}_{2} \mathrm{His}_{2}$ Zinc Finger Proteins. Annu Rev Biophys Biomol Struct 29: 183-212.

9. Li H, Haurigot $\mathrm{V}$, Doyon Y, Li T, Wong SY, et al (2011) In vivo genome editing restores haemostasis in a mouse model of haemophilia. Nature 475: 217-221.

10. Meng X, Noyes MB, Zhu LJ, Lawson ND, Wolfe A (2008) Targeted gene inactivation in zebrafish using engineered zinc-finger nucleases. Nat Biotechnol 26: 695-701.
11. Boch J, Scholze H, Schornack S, Landgraf A, Hahn S (2009) Breaking the code of DNA binding. Science 326: 1509-1512.

12. Horvath P, Barrangou R (2010) CRISPR/Cas, the immune system of bacteria and archaea. Science 327: 167-170.

13. Ding Q, Lee Y, Schaefer EAK, Peters DT, Veres A, et al. (2014) A TALEN genome-editing system for generating human stem cell-based disease models. J Stem 12: 238-251.

14. Basu S, Sachidanandan C (2013) Zebrafish: A multifaceted tool for chemical biologists. Chem Rev 113: 7952-7980.

15. Yang L, Esvelt KM, Aach J, Guell M, James E, et al. (2013) RNA-guided human genome engineering via Cas9. Science 339: 823-826.

16. Grissa I, Vergnaud G, Pourcel C (2007) The CRISPRdb database and tools to display CRISPRs and to generate dictionaries of spacers and repeats. BMC Bioinformatics 10: 1-10.

17. Ishino Y, Shinagawa H, Makino K, Amemura M, Nakata A (1987) Nucleotide sequence of the iap gene, responsible for alkaline phosphatase isozyme conversion in Escherichia coli and identification of the gene product. J Bacteriol 169: 5429-5433.

18. Broadbent JR, Neeno-Eckwall CE, Stahl B, Tandee K, Cai H, et al. (2012) Analysis of the Lactobacillus casei supragenome and its influence in species evolution and lifestyle adaptation. BMC Genomics 13: 533.

19. Shui B, Matias LH, Guo Y, Peng Y (2016) The rise of CRISPR/Cas for genome editing in stem cells. Stem Cells Int 2016: 1-17.

20. Van der Oost J, Jore MM, Westra ER, Lundgren M, Brouns SJJ (2009) CRISPRbased adaptive and heritable immunity in prokaryotes. Trends Biochem Sci 34: 401-407.

21. Deltcheva E, Chylinski K, Sharma CM, Gonzales K (2011) CRISPR RNA maturation by trans-encoded small RNA and host factor RNase III. 471: 602607.

22. Jinek M, Chylinski K, Fonfara I, Hauer M, Jennifer A, et al. (2012) Doudna: A programmable dual-RNA-guided DNA endonuclease in adaptive bacterial immunity. Science 337: 816-821.

23. Haft DH, Selengut J, Mongodin EF, Nelson KE (2005) A guild of 45 CRISPRassociated (Cas) protein families and multiple CRISPR/Cas subtypes exist in prokaryotic genomes. PLoS Comput Biol 1: e60.

24. Bassing CH, Alt FW (2004) The cellular response to general and programmed DNA double strand breaks. DNA Repair 3: 781-796.

25. Pourcel C, Salvignol G, Vergnaud G (2005) CRISPR elements in Yersinia pestis acquire new repeats by preferential uptake of bacteriophage DNA and provide additional tools for evolutionary studies. Microbiology 151: 653-663.

26. Mojica FJM, Díez-Villaseñor C, García-Martínez J, Soria E (2005) Intervening sequences of regularly spaced prokaryotic repeats derive from foreign genetic elements. J Mol Evol 60:174-182.

27. Swarts DC, Mosterd C, Passel MWJ Van, Brouns SJJ (2012) CRISPR interference directs strand specific spacer acquisition. PLoS One 7: e35888.

28. Burgess DJ (2014) In vivo correction of genetic disease in adult mice. Nat Rev Genet 15: 291-291.

29. Burgess DJ (2014) Technology: A CRISPR genome-editing tool. Nat Biotech 32: 347-355.

30. Saayman S, Ali SA, Morris KV, Weinberg MS (2015) The therapeutic application of CRISPR/Cas9 technologies for HIV. Exp Opin Biol Ther 15: 819-830.

31. Manuscript A (2014) NIH Public Access. 153: 910-918.

32. Yang H, Wang H, Shivalila CS, Cheng AW, Shi L, et al. (2013) Resource onestep generation of mice carrying reporter and conditional alleles by CRISPR/ Cas-mediated genome engineering. Cell 154: 1370-1379.

33. Grissa I, Vergnaud G, Pourcel C (2007) CRISPRFinder : A web tool to identify clustered regularly interspaced short palindromic repeats. Nucleic Acids Res 35: W52-W57.

34. Rath D, Amlinger L, Rath A, Lundgren M (2015) The CRISPR-Cas immune system: Biology, mechanisms and applications. Biochimie 117: 119-128.

35. Anders C, Niewoehner O, Duerst A, Jinek M (2015) Structural basis of PAMdependent target DNA recognition by the Cas 9 endonuclease. Nature 513 : 569-573. 
36. Jinek M, Jiang F, Taylor DW, Sternberg SH, Kaya E, et al. (2014) Structures of Cas9 endonucleases reveal RNA-mediated conformational activaztion. Science 343: 1-28.

37. Nishimasu H, Ran FA, Hsu PD, Konermann S, Shehata SI, et al. (2014) Crystal structure of Cas9 in complex with guide RNA and target DNA. Cell 156: 935949.

38. Fremaux C, Horvath $P(2010)$ The CRISPR/Cas bacterial immune system cleaves bacteriophage and plasmid DNA. Nature 468: 67-71.

39. Wiedenheft B, Duijn EV, Bultema JB, Waghmare SP, Zhou K, et al. (2011) RNAguided complex from a bacterial immune system enhances target recognition through seed sequence interactions. PNAS 108: 10092-10097.

40. Ousterout GD, Kabadi MA, Thakore IP, Majoros HW, Reddy ET, et al. (2015) Multiplex CRISPR/Cas9-based genome editing for correction of dystrophin mutations that cause Duchenne muscular dystrophy. Nat Commun 6: 6244.

41. Puchta $H$ (2017) The repair of double-strand breaks in plants: Mechanisms and consequences for genome evolution. J Exp Bot 56: 1-14.

42. Hsu PD, Scott DA, Weinstein JA, Ran FA, Konermann S, et al. (2013) DNA targeting specificity of RNA-guided Cas9 nucleases. Nat Biotechnol 31: 827832.

43. Mali P, Aach J, Stranges PB, Esvelt KM, Moosburner M, et al. (2013) CAS9 transcriptional activators for target specificity screening and paired nickases for cooperative genome engineering. Nat Biotechnol 31: 833-838.

44. Nuclease RC, Gratz SJ, Cummings AM, Nguyen JN, Hamm DC, et al. (2013) Genome engineering of drosophila with the CRISPR. Genetics 194: 1029-1035.

45. Schiml S, Fauser F, Puchta H (2014) The CRISPR/Cas system can be used as nuclease for in planta gene targeting and as paired nickases for directed mutagenesis in Arabidopsis resulting in heritable progeny. Plant J 9: 1139-1150.

46. Shukla VK, Doyon Y, Miller JC, DeKelver RC, Moehle EA, et al. (2009) Precise genome modification in the crop species Zea mays using zinc-finger nucleases. Nature 459: 437-441.

47. Gratz SJ, Ukken FP, Rubinstein CD, Thiede G, Donohue LK, et al. (2014) Highly specific and efficient CRISPR/Cas9-catalyzed homology-directed repair in drosophila. Genetics 196: 961-971.

48. Ding Y, Li H, Chen L, Xie K, Chen L (2016) Recent advances in genome editing using CRISPR/Cas9. Front Plant Sci 7: 1-12

49. Thomas CE, Ehrhardt A, Kay MA (2003) Progress and problems with the use of viral vectors for gene therapy. Nat Rev Genet 4: 346-358

50. Anson DS (2004) The use of retroviral vectors for gene therapy-what are the risks ? A review of retroviral pathogenesis and its relevance to retroviral vectormediated gene delivery. Genet Vaccines Ther 13: 1-13.

51. Mingozzi F, High KA (2011) Therapeutic in vivo gene transfer for genetic disease using AAV: Progress and challenges. Nat Rev Genet 12: 341-355.

52. Mali P, Esvelt KM, Church GM (2013) Cas9 as a versatile tool for engineering biology. Nat Methods 10: 957-963.

53. Horvath P, Romero DA, Cou A, Richards M, Moineau S, et al. (2008) Diversity, activity and evolution of CRISPR loci in Streptococcus thermophilus. J Bacteriol 190: 1401-1412.

54. Barrangou R, Garneau JE, Labonte J, Fremaux C, Boyaval P, et al. (2008) Phage response to CRISPR-encoded resistance in Streptococcus thermophilus. J Bacteriol 190: 1390-1400.

55. Zetsche B, Gootenberg SJ, Abudayyeh OO, Slaymaker IM, Makarovaet SK, et al. (2015) Cpf1 is a single RNA-guided endonuclease of a Class 2 CRISPRCas system. Cell 520: 186-191.

56. Naldini L, Blömer U, Gallay P, Ory D, Mulligan R, et al. (1996) In vivo gene delivery and stable transduction of nondividing cells by a lentiviral vector. Science 272: 263-267.

57. Yin H, Xue W, Chen S, Bogorad RL, Benedetti E, et al. (2014) Genome editing with Cas9 in adult mice corrects a disease mutation and phenotype. Nat Biotechnol 32: 551-553.

58. Hough SH, Ajetunmobi A (2017) The future of CRISPR applications in the lab, the clinic and society. In: Tsang S. (eds) Precision medicine, CRISPR and genome engineering. Adv Exp Med Biol 1016: 157-178.

59. Manuscript A, Delivery HG (2011) NIH Public Access. 28: 694-701.

60. Qi LS, Larson MH, Gilbert LA, Doudna JA, Weissman JS, et al. (2013) Resource repurposing CRISPR as an RNA-guided platform for sequence-specific contro of gene expression. Cell 152: 1173-1183.

61. Qi LS, Larson MH, Gilbert LA, Doudna JA, Weissman JS, et al. (2013) Repurposing CRISPR as an RNA-guided platform for sequence-specific control of gene expression. Cell 152: 1173-1183.

62. Song G, Jia M, Chen K, Kong X, Khattak B, et al. (2016) CRISPR/Cas9: A powerful tool for crop genome editing. Crop J 4: 75-82.

63. Chen B, Gilbert LA, Cimini BA, Schnitzbauer J, Zhang W, et al. (2013) Resource dynamic imaging of genomic loci in living human cells by an optimized CRISPR/ Cas system. Cell 155: 1479-1491.

64. Zetsche B, Gootenberg JS, Abudayyeh OO, Ian M, Oost JVD, et al. (2016) Crystal structure of crispr cpf. Cell 163: 759-771.

65. Lander ES (2015) Perspective the heroes of CRISPR. Cell 164: 18-28.

66. Wang H, Yang H, Shivalila CS, Dawlaty MM, Cheng AW, et al. (2013) Resource one-step generation of mice carrying mutations in multiple genes by CRISPR/ Cas-mediated genome engineering. Cell 153: 910-918.

67. Lin Y, Cradick JT, Brown TW, Deshmukh H, Ranjan R, et al. (2014) CRISPR Cas9 systems have off-target activity with insertions or deletions between target DNA and guide RNA sequences. Nucleic Acids Res 42: 7473-7485.

68. Ebina H, Misawa N, Kanemura Y, Koyanagi Y (2013) Harnessing the CRISPR/ Cas9 system to disrupt latent HIV-1 provirus. Sci Rep 3: 2510.

69. Schwank G, Koo B, Sasselli V, Dekkers JF, Heo I, et al. (2013) Brief report functional repair of CFTR by CRISPR/Cas 9 in intestinal stem cell organoids of cystic fibrosis patients. Stem Cell 13: 653-658.

70. Yui S, Nakamura T, Sato T, Nemoto Y, Mizutani T, et al. (2012) Functional engraftment of colon epithelium expanded in vitro from a single adult lgr5+ stem cell. Nature Med 18: 618-623.

71. Lisa $\mathrm{H}$, Gee $\mathrm{P}$, Ishida $\mathrm{K}$, Hotta $A$ (2016) Efficient genomic correction methods in human iPS cells using CRISPR-Cas9 system. Methods 101: 27-35.

72. Wang P, Mokhtari R, Pedrosa E, Kirschenbaum M, Bayrak C, et al. (2017) CRISPR/Cas9-mediated heterozygous knockout of the Autism gene CHD8 and characterization of its transcriptional networks in cerebral organoids derived from iPS cells. Mol Autism Brain Cogn Behav 8: 11.

73. Ricciardi S, Ungaro F, Hambrock M, Rademacher N, Stefanelli G, et al. (2012) CDKL5 ensures excitatory synapse stability by reinforcing NGL-1-PSD95 interaction in the postsynaptic compartment and is impaired in patient iPSCderived neurons. Nat Cell Biol 14: 911-923.

74. Lett C (2017) HHS public access. 373: 109-118.

75. Hu Z, Yu L, Zhu D, Ding W, Wang X, et al. (2014) Disruption of HPV16-E7 by CRISPR/Cas system induces apoptosis and growth inhibition in HPV16 positive human Cervical Cancer cells. BioMed Res Int 2014: 1-9.

76. Liang X, Sun L, Yu T, Pan Y, Wang D, et al. (2016) A CRISPR/Cas9 and Cre/ Lox system-based express vaccine development strategy against re-emerging Pseudorabies virus. Nat Publ Gr 2015: 1-10.

77. Wang G, Zhao N, Berkhout B, Das AT, Wang G, et al. (2016) Report a combinatorial CRISPR-Cas9 attack on HIV-1 DNA extinguishes all infectious provirus in infected T cell cultures. Cell Rep 17: 2819-2826.

78. Pham EA, Perumpail RB, Fram BJ, Glenn JS, Ahmed A, et al. (2016) Future therapy for Hepatitis B virus: Role of immunomodulators. Curr Hepatol Reports 2016: 237-244.

79. Feng Z, Mao Y, Xu N, Zhang B, Wei P, et al. (2014) Multigeneration analysis reveals the inheritance, specificity and patterns of CRISPR/Cas-induced gene modifications in Arabidopsis. PNAS 111: 4632-4637.

80. Jiang W, Zhou H, Bi H, Fromm M, Yang B, et al. (2013) Demonstration of CRISPR/Cas9/sgRNA-mediated targeted gene modification in Arabidopsis, tobacco, sorghum and rice. Nucleic Acids Res 41: e188.

81. Auer TO, Duroure K, Cian A De, Concordet J, Bene F (2014) Highly efficient CRISPR/Cas9-mediated knock-in in zebrafish by homology-independent DNA repair. Genome Res 24: 142-153.

82. Bassett AR, Tibbit C, Ponting CP, Liu J (2014) Correction highly efficient targeted mutagenesis of Drosophila with the CRISPR/Cas9 system target site. Cell Rep 6: 1178-1179.

83. Sander DJ, Joung KJ (2014) CRISPR-Cas systems for editing, regulating and targeting genomes. Nat Biotechnol 32: 347-355. 
84. Xie K, Yang Y(2013) RNA-Guided genome editing in plants using a CRISPRCas system. Mol Plant 6: 1975-1983.

85. http://www.stem-art.com/Library/Science/Cell\%20Reports\%20best\%20of\%20 2014.pdf

86. Cheng AW, Wang H, Yang H, Shi L, Katz Y, et al. (2013) Multiplexed activation of endogenous genes by CRISPR-on, an RNA-guided transcriptional activator system. Nat Publ Gr 23: 1163-1171.

87. Arora L, Narula A (2017) Gene editing and crop improvement using CRISPRCas9 system. Front Plant Sci 8: 1932.

88. https://stacks.cdc.gov/view/cdc/30029/cdc_30029_DS1.pdf

89. https://stacks.cdc.gov/view/cdc/30309/cdc_30309_DS1.pdf

90. Xiao A, Cheng Z, Kong L, Zhu Z, Lin S, et al. (2017) CasOT: A genome-wide Cas9/gRNA off-target searching tool. Bioinformatics 30: 1180-1182.

91. Xie K, Zhang Y, Yang Y(2014) Genome-wide prediction of highly specific guide
RNA spacers for CRISPR-Cas9-mediated genome editing in model plants and major crops. Mol Plant 7: 923-926.

92. Shan Q, Wang Y, Li J, Gao C (2014) Genome editing in rice and wheat using the CRISPR/Cas system. Nat Protoc 18: 2395.

93. Karvelis T, Gasiunas G, Miksys A, Barrangou R, Horvath P, et al. (2013) crRNA and tracrRNA guide Cas9-mediated DNA interference in Streptococcus thermophilus. RNA Biol 10: 841-851.

94. Garneau JE, Dupuis MĖ, Villion M, Romero DA, Barrangou R, et al. (2010) The CRISPR/Cas bacterial immune system cleaves bacteriophage and plasmid DNA. Nature 468: 67-71.

95. Zhang Y, Heidrich N, Ampattu JB, Gunderson WC, Seifert SH, et al. (2013) Processing-independent CRISPR RNAs limit natural transformation in Neisseria meningitidis. Mol Cell 50: 488-503.

96. Hou Z, Zhang Y, Propson NE, Howden SE, Chu LF, et al. (2013) Efficient genome engineering in human pluripotent stem cells using Cas9 from Neisseria meningitidis. Proc Natl Acad Sci 110: 15644-15649. 